\title{
EFFICIENT IMPLEMENTATION OF CDP ALGORITHMS FOR OPTIMAL EVALUATION OF ARRAY EXPRESSIONS
}

m.f abdulla, ks alWagih

\begin{abstract}
Executing array expressions on massively parallel machines can suffer considerable slow due to the heavy communication between the processors. Such communications can significantly be reduced with the optimal mapping of the array operations to the processors in the system. This mapping can be done either by the selected language compiler or by the programmer himself. In this work, we investigate compact dynamic programming (CDP) methods [1,3] to get optimal mapping of the array operations to the physical processors in the system under the data parallelism paradigm. We address this problem through implementing different CDP algorithms for a variety of the interconnected schemes of the processors, viz, grids, rings, discrete, and FAT metric. The evaluated results are very encouraging. The optimizing algorithms implemented in $\mathrm{C}++$ compiler. We provide a way to reduce the complexity of CDP for certain trees.
\end{abstract}

